\title{
CRIOGLOBULINEMIA MIXTA ESENCIAL TIPO II: PRESENTACIÓN DE UN CASO.
}

ESSENTIAL MIXED CRYOGLOBULINEMIA TYPE II: CASE REPORT.

\author{
Marco Mazzotta ${ }^{1}$, Beatriz Dotto ${ }^{2}$, Viviana Zalazar ${ }^{3}$, Mónica Herrero $^{4}$, Ana María Sesin ${ }^{2}$, María Elisa \\ Dionisio de Cabalier ${ }^{1}$, Jorge Mukdsi, ${ }^{1,5}$.
}

1 Cátedra de Anatomía Patológica y Servicio de Anatomía Patológica-FCM-UNC. Argentina.

2 Servicio de Nefrología-Hospital Nacional de Clínicas. FCM-UNC. Argentina.

3 Cátedra de Dermatología- Hospital Nacional de Clínicas. FCM-UNC. Argentina.

4 Laboratorio de Privado de Patología. Córdoba. Argentina.

5 Email de contacto: mukdsijorge@gmail.com

Resumen:

El síndrome crioglobulinémico es producido por inmunoglobulinas que precipitan reversiblemente a bajas temperaturas, llamadas crioglobulinas. Su producción se asocia a varias causas, como desórdenes linfoproliferativos, infecciones crónicas y trastornos autoinmunitarios. No obstante, en muchos casos la etiología no logra determinarse. Existen tres tipos, siendo las de tipo II y III las que forman parte de las denominadas crioglobulinemias mixtas. Estas últimas producen un cuadro de vasculitis sistémica con manifestaciones clínicas diversas. La púrpura palpable es la más frecuente, acompañada en forma variable por artralgias, neuropatía y afección renal en forma de glomerulonefritis membranoproliferativa de tipo I. Presentamos el caso clínico de un paciente de sexo masculino de 71 años de edad con insuficiencia renal, síndrome nefrítico, hipertensión arterial y lesiones cutáneas purpúricas palpables ambos miembros, tórax y abdomen. Durante la internación se diagnostica crioglobulinemia mixta esencial asociada a vasculitis leucocitoclástica cutánea y glomerulonefritis membranoproliferativa tipo I. Describimos los métodos complementarios utilizados para llegar al diagnóstico, el tratamiento instituido y la evolución clínica del paciente.

Palabras clave: crioglobulinemia esencial; vasculitis leucocitoclástica; glomerulonefritis.

\section{Abstract:}

The cryoglobulinemic syndrome is produced by precipitating immunoglobulins at low temperatures. Its production is associated with several causes, such as lymphoproliferative disorders, chronic infections and autoimmune disorders. However, the etiology is unknow. There are three types of cryoglobulins. Type II and III are the mixed. Type III produce a systemic vasculitis with diverse clinical manifestations. Palpable purpura is the most common, accompanied by arthralgia, neuropathy and type I membranoproliferative glomerulonephritis. We present a case of a 71 years old male patient with renal failure, nephritic syndrome, arterial hypertension and palpable purpuric skin lesions in members, thorax and abdomen. During hospitalization essential mixed cryoglobulinemia associated with cutaneous leukocytoclastic vasculitis and type I membranoproliferative glomerulonephritis was diagnosed. Complementary methods, treatment instituted and the patient's clinical course are described.

Keywords: mixed cryoglobulinemia; cryoglobulinaemic vasculitis; glomerulonephritis. 


\section{Introducción}

Las crioglobulinas ( $\mathrm{CrG}$ ) son inmunoglobulinas que precipitan reversiblemente a bajas temperaturas. La existencia de CrG circulantes no siempre se asocia a la presencia de síntomas, y el concepto de síndrome crioglobulinémico se aplica cuando los pacientes con CrG presentan manifestaciones clínicas. Se han descripto tres tipos de CrG: tipo I (monoclonales), tipo II (IgM monoclonales con actividad anti-lgG); y tipo III (IgG e IgM policlonales) ${ }^{1}$. Las de tipo II y III son las más comunes y forman parte de las llamadas crioglobulinemias mixtas (CM).

La vasculitis crioglobulinémica (VC) es producida por depósitos de CrG en pequeños vasos (predominantemente capilares, vénulas o arteriolas) y está asociada a CrG séricas ${ }^{2}$. Asimismo, se ha descrito afección de vasos musculares de calibre mediano en una minoría de casos $^{3}$.

Las CrG tipo I se asocian con frecuencia a trastornos linfoproliferativos, en tanto las CM a infecciones crónicas, trastornos linfoproliferativos y/o enfermedades autoinmunes. En un $5 \%$ a $10 \%$ de pacientes no se consigue identificar etiología alguna, constituyendo la CM esencial ${ }^{4-6}$.

\section{Caso clínico}

Paciente de sexo masculino de 71 años de edad con antecedente de hipertensión arterial (HTA) que consulta por presentar edema en miembros inferiores de localización infrapatelar, disnea y astenia de un mes de evolución. Presentaba púrpura palpable en miembros superiores e inferiores, tórax y abdomen (Figura $1 \mathrm{~A}$ ). Al ingreso, los estudios de laboratorio demostraron: hematocrito 29\%, hemoglobina 9,5 g/dl, glóbulos blancos $6.900 / \mathrm{mm}^{3}$, plaquetas $93.000 / \mathrm{mm}^{3}$, glucosa $101 \mathrm{mg} / \mathrm{dl}$, creatinina $2,19 \mathrm{mg} / \mathrm{dl}$. En el sedimento urinario se evidenciaron células epiteliales 0,5/campo, leucocitos 8/campo, hematíes +100/campo, cilindros hialinos 7/preparado, cilindros hialino-hemáticos 1/preparado, cilindros hemáticos 2/preparado, hematíes dismórficos y proteinuria de 1,2 g/12 h. Durante la internación se le realizó biopsia cutánea. En la dermis subyacente se evidenciaron signos de vasculitis leucocitoclástica con afección de la totalidad de los vasos del plexo capilar superficial y profundo (Figura 1 B y C). La función renal del paciente empeoró lenta y paulatinamente presentando además HTA de 190/100 mmHg. Se instauró terapia con amlodipina (10 mg/12 h), enalapril (20 mg/12 h), losartán (50 mg/12 h), espironolactona (100 $\mathrm{mg} /$ día), furosemida $(20 \mathrm{mg} / 6 \mathrm{~h})$ y atenolol $(12,5 \mathrm{mg} / 12 \mathrm{~h})$, sin poder llevar los valores dentro de parámetros normales. Por confluencia de las lesiones cutáneas, presencia de dolor en las mismas y empeoramiento gradual de la función renal se decide comenzar con tratamiento corticoideo (prednisona $60 \mathrm{mg} /$ día). Al día siguiente se inicia un ciclo de metilprednisolona EV de $1 \mathrm{~g} /$ día y de $500 \mathrm{mg} / \mathrm{día}$ los 4 días posteriores, continuando después con $60 \mathrm{mg} / \mathrm{día}$ de prednisona vía oral.

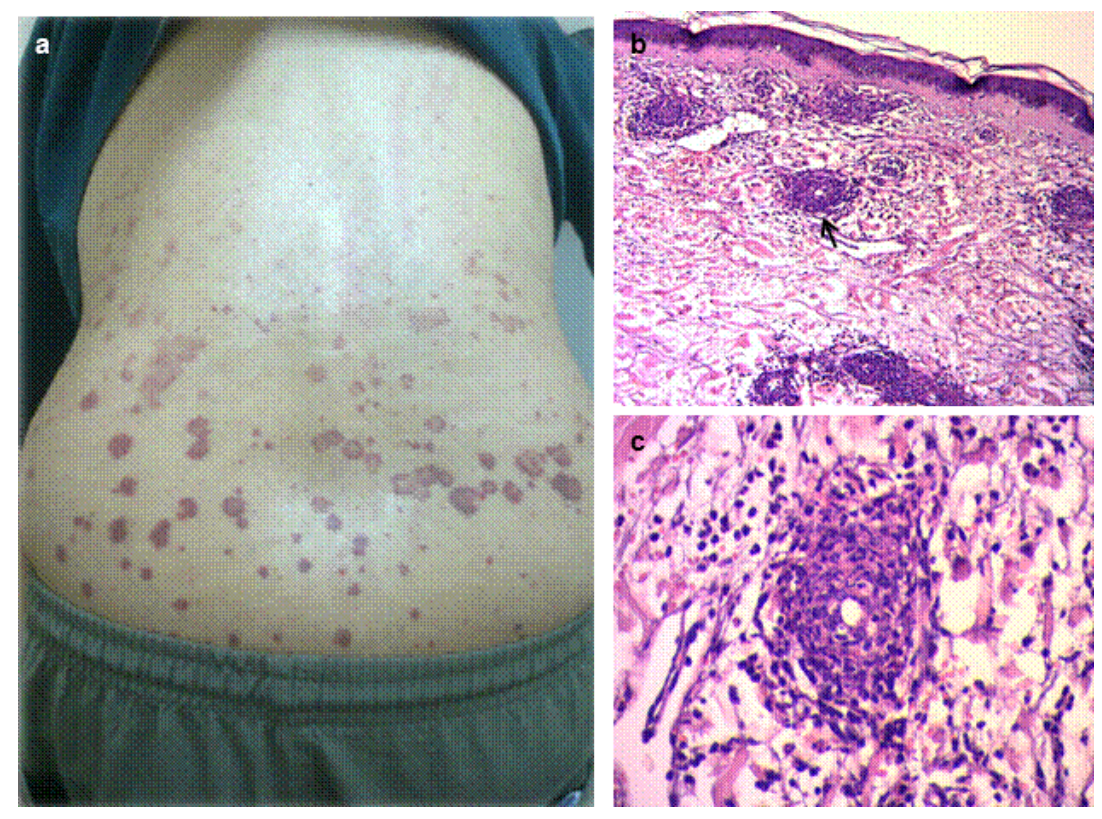

Figura 1: A-Lesiones purpúricas en tronco. B-Vasculitis aguda leucocitoclástica Se aprecia infiltrado de polimorfosnucleares neutrófilos, acompañados por un menor número de linfocitos y aislados eosinófilos con moderada cariorexis y por extravasación de eritrocitos (Hematoxilina/Eosina. 100X). C-El vaso afectado presenta tumefacción endotelial y disminución de su luz (Hematoxilina/Eosina. 400X). 
Al momento de comenzar la corticoterapia el paciente presentaba creatinina de 2,96 mg/dl, llegando a ascender hasta $3,11 \mathrm{mg} / \mathrm{dl}$. Posteriormente este parámetro de función renal comenzó en franco descenso con mejoría clínica de las lesiones dérmicas, desapareciendo por completo a los 3 días de administración de metilprednisolona.

Se decide realizar biopsia renal. La microscopia óptica demostró dos estructuras glomerulares con marcada lobulación del penacho capilar, aumento de celularidad endotelial y mesangial y engrosamiento de membranas basales glomerulares (Figura 2 A). Se identificaron signos de daño tubular agudo y moderada cantidad de cilindros hemáticos intraluminales. La inmunofluorescencia directa demostró inmunorreactividad para IgM (+++) e IgG $(+++)$, cadenas kappa y lambda positivas (+) (Figura 2 B). El estudio ultraestructural mostró la presencia de numerosas células mononucleares y escasos polimorfonucleares neutrófilos que ocupaban los lúmenes capilares. Los primeros presentaban numerosas vesículas de tipo lisosomal con marcada electrodensidad (Figura $2 \mathrm{C}$ ). Se identificaron numerosos depósitos electrodensos del tipo de complejos inmunes, de diversos tamaños, en espacio subendotelial y que a grandes magnificaciones evidenciaron una organización microtubular (Figura 2 D). Se observó engrosamientos de las membranas basales glomerulares a expensas de la interposición de citoplasmas entre las mismas y el endotelio subyacente. No se identificaron inclusiones túbulo-reticulares en el citoplasma de las células endoteliales.
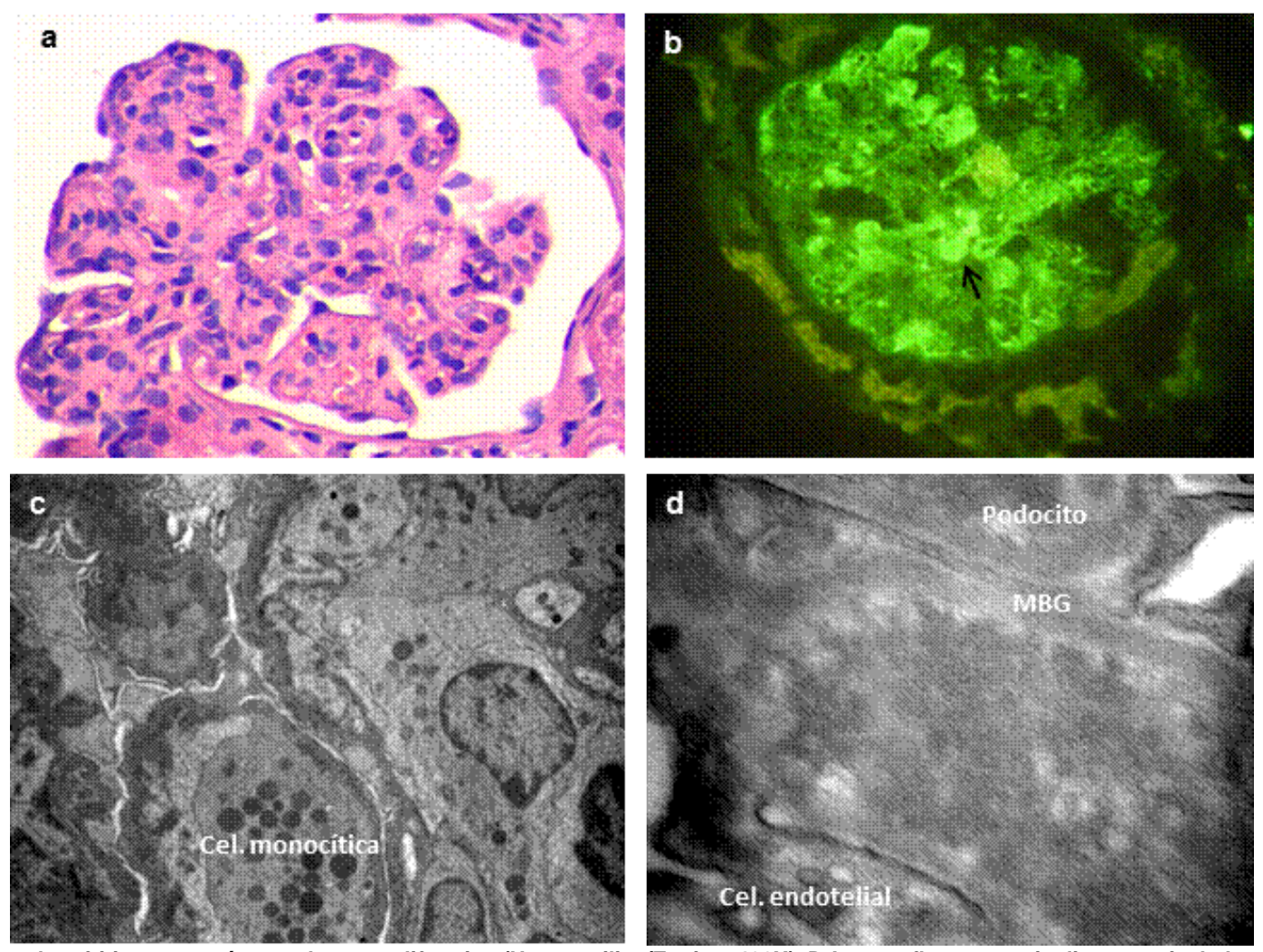

Figura 2: C-Glomerulonefritis con patrón membranoproliferativo (Hematoxilina/Eosina. 400X). D-Inmunofluorescencia directa a nivel glomerular. Nótese la intensa inmunoreactividad para IgG trombos proteicos intraluminales (400X). D-Microfotografía electrónica de transmisión que muestra células monocíticas con marcado desarrollo del sistema endocítico-fagocítico (7000X). E-Depósitos organizados conformando estructuras microtubulares subendoteliales (30000X).

La serología viral demostró: anticuerpos (Ac) anti-VIH: no reactivo; Ac anti-HBc (core) total: 5,96-reactivo (valor normal hasta 0,50); Ag-HBs: no reactivo; Ac anti-VHC: no reactivo. Complemento: C3: 108 (valor de referencia: $80-180 \mathrm{mg} \%$ ) y C4 no dosable (valor de referencia: $15-40 \mathrm{mg} \%$ ). Crioglobulinas positivas y factor reumatoideo $1 / 10240$.

El diagnóstico final fue de CM esencial de tipo II asociada a vasculitis leucocitoclástica y glomerulonefritis membranoproliferativa (GNMP).

Al alta del paciente se indica: terapéuticas al alta del paciente: ranitidina (300 mg/día), amlodipina (10 $\mathrm{mg} / 12 \mathrm{~h}$ ), losartán (50 mg/12 h), espironolactona (50 mg/12 h), furosemida (40 mg/6 h), enalapril (10 $\mathrm{mg} / 12 \mathrm{~h}$ ), atenolol (12,5 mg/12 h), pregabalina (50 mg/día). 
Un mes posterior a la primera consulta se diagnostica neumopatía infecciosa aguda e insuficiencia renal crónica reagudizada. Fue medicado con levofloxacina $500 \mathrm{mg}$ (1/día x 6 días/48 h), prednisona, furosemida, amlodipina, losartán (todos a las mismas dosis que las anteriores) y omeprazol (40 mg/día). Presentó buena respuesta al tratamiento instituido y se le otorgó el alta hospitalaria.

Un mes después, el paciente fue admitido nuevamente por un cuadro de lipotimia, malestar general y síndrome febril. La radiografía de tórax reveló infiltrado alvéolo-intersticial-nodular en zona media de campo pulmonar derecho, base derecha y vértice izquierdo. El diagnóstico fue de neumopatía infecciosa aguda bilateral. Se realizó fibrobroncoscopía la cual mostró marcada congestión del árbol traqueobronquial. Se instauró tratamiento antibiótico empírico de amplio espectro ante la sospecha de probable neumonía intrahospitalaria: piperacilina-tazobactam $(2,25 \mathrm{~g} / 6 \mathrm{~h})+$ vancomicina $(1 \mathrm{~g} / 48 \mathrm{~h})$, metronidazol vía oral (1 comprimido cada 8 h). Tras un mes de internación el paciente fue trasladado a la unidad de cuidados intensivos por presentar insuficiencia respiratoria aguda. Se le otorgó asistencia respiratoria mecánica más un plan de hidratación, antibióticos de amplio espectro, protección gástrica, antiinflamatorios y anticoagulantes. Al tercer día el paciente falleció debido a un choque séptico con disfunción multiorgánica secundario a neumonía intrahospitalaria.

\section{Discusión}

La crioglobulinemia es una condición patológica causada por la producción excesiva de inmunoglobulinas circulantes que precipitan por el frío y se redisuelven con el calentamiento, y se presenta como un síndrome clínico de inflamación sistémica que afecta principalmente la piel y riñones ${ }^{7}$. El caso que presentamos corresponde a una CM esencial por tener un componente de $\operatorname{lgM}$ con actividad de factor reumatoideo y sin identificación de etiología alguna. Debemos resaltar que los tipos II y III de crioglobulinas mixtas son inmunocomplejos compuestos por lgGs policlonales que son autoantígenos y las IgMs son monoclonales o policlonales, respectivamente, siendo las IgMs los autoanticuerpos con actividad de factor reumatoide ${ }^{6,7}$.

La demostración patológica de inflamación en las paredes vasculares es el método más apropiado y definitivo para hacer el diagnóstico de vasculitis. Las dos categorías inmunopatológicas principales de las vasculitis son aquellas con depósitos de inmunoglobulinas, entre las que se encuentran las por CrG y aquellas con poco o nada de depósitos inmunes en la pared vascular y que son denominadas pauciinmunes ${ }^{2}$.

Considerando el caso presentado podemos decir que el mismo cumple con criterios mayores y menores tanto serológicos, clínicos y patológicos para ser diagnosticado como una $\mathrm{VC}^{8}$.

Serológicamente, se detectó en el paciente una hipocomplementemia a expensas de la fracción C4, hallazgo útil y característico de esta patología. El patrón clínico de las VC es comparable en las CM tipo II y $1 \mathrm{II}^{8}$, siendo las lesiones cutáneas las manifestaciones más frecuentes de las $\mathrm{CM}^{9}$. Las CrG producen síntomas debido a la obstrucción de los pequeños vasos, particularmente en las extremidades (que están más expuestas al frío) o por depósito de complejos inmunes en las paredes vasculares ${ }^{10}$. La púrpura palpable recurrente con hiperpigmentación postinflamatoria es la presentación clínica más frecuente, aunque también se han descrito nódulos, úlceras, livedo racemosa y gangrena digital como expresión de vasculitis de vasos musculares de mediano calibre. La extensión y severidad de las lesiones aumenta en ausencia de un adecuado tratamiento, tal como sucedió en este caso ${ }^{3}$.

Refiriéndose a los criterios anatomopatológicos, las biopsias cutánea y renal realizadas en este paciente demostraron una vasculitis leucocitoclástica y un cuadro de glomerulonefritis membranoproliferativa (GNMP) tipo I, respectivamente. La vasculitis leucocitoclástica es la marca de las $\mathrm{CM}^{8,11}$ y es fácil de demostrar por medio de la biopsia de lesiones cutáneas recientes, usualmente dentro de las primeras 48 horas $^{12}$. Histológicamente hay una inflamación angiocéntrica segmentaria con tumefacción de las células endoteliales, necrosis fibrinoide de las paredes de los vasos y un infiltrado compuesto sobre todo por polimorfonucleares que muestran fragmentación del núcleo (cariorrexis o leucocitoclasia) ${ }^{13}$. La afección es de vasos pequeños tanto de la dermis superficial y profunda como de hipodermis, con involucro de vasos musculares en un pequeño porcentaje de $\operatorname{casos}^{3}$. Las lesiones serían causadas por el depósito de complejos inmunes en las vénulas poscapilares, activando al complemento y mediadores de la inflamación 
(leucotrieno B4, histamina, trombina, IL-1, IL-6, FNT-alfa e interferones) que contribuyen al daño endotelial $^{14}$.

En relación a la glomerulonefritis crioglobulinémica (GNCG), esta es un tipo de GN mediada por complejos inmunes, aunque otros mecanismos patogénicos han sido también propuestos, presentándose hasta en el $20 \%$ de los casos de pacientes con crioglobulinemia ${ }^{15}$. La nefropatía crioglobulinémica ocurre casi exclusivamente asociada a CM tipo II donde se detecta una IgM circulante ${ }^{16}$. La GNMP tipo 1 es la forma histológica más comúnmente observada en la $\mathrm{CM}$, siendo la forma de presentación más habitual la proteinuria y la microhematuria, pudiendo también existir insuficiencia renal moderada ${ }^{17}$.

Aunque por microscopía fotónica las lesiones glomerulares de la VC pueden mostrar una variedad de características proliferativas o esclerosantes, ciertos rasgos ayudan a distinguir las GN proliferativas de la CM esencial de otras GN proliferativas.

Los monocitos de los pacientes con crioglobulinemia activa y nefritis asociada a la misma han mostrado CrG fagocitadas pero que son incapaces de ser catabolizadas por ellos. Tal como se describe a nivel glomerular, en el presente caso se apreciaron numerosas células monocíticas asociadas a depósitos subendoteliales, además de las clásicas manifestaciones morfológicas de la GNMP tipo ${ }^{18}$. Los diagnósticos diferenciales de las GNCG incluyen no solo las formas primarias de GNMP, sino también otras formas de vasculitis sistémicas, las microangiopatías trombóticas, la rara macroglobulinemia de Waldenström y el lupus eritematoso sistémico. Para esta última situación la cuidadosa evaluación de C1q por IF debería conducir al diagnóstico de GN lúpica. En nuestro caso una herramienta de gran valor fue el estudio ultraestructural, donde los depósitos subendoteliales e intraluminales demostraron la específica estructura microtubular descripta en los casos de $\mathrm{GNCG}^{19,20}$, además de la presencia de numerosas células de naturaleza monocítica con marcado desarrollo del sistema endosómico-lisomal.

En concordancia con el hecho de que la $\operatorname{lgG}$ es el tipo más común de crioglobulina en los depósitos detectados por IFD, es esta lg la que predomina, habiéndose descripto tres patrones de inmunomarcación ${ }^{21}$. En un $92 \%$ de los casos también se inmunodetecta IgM y $\mathrm{C3}^{22}$.

Con respecto a la causa de las $\mathrm{CM}$, debemos tomar como referencia el descubrimiento del virus de la hepatitis $\mathrm{C}(\mathrm{VHC})^{23}$. Después de este acontecimiento científico, varios reportes informaron una asociación entre virus de la hepatitis $\mathrm{C}$ y la $\mathrm{CM}$ en un 80 a $90 \%$ de los $\operatorname{casos}^{24,25}$. Menos del $5 \%$ de los pacientes con $\mathrm{CrG}$ tienen asociación puramente con $\mathrm{VHB}^{26,27}$. El rol del VHB ha sido investigado, sin embargo la viremia raramente se encuentra ${ }^{28}$, mientras que los niveles de anticuerpos contra VHB varían entre las diferentes poblaciones de pacientes con $\mathrm{CM}^{8}$. En 1977 fue la primera vez que se sugirió tal asociación ${ }^{28}$ pero estudios posteriores ofrecieron resultados conflictivos ${ }^{25}$. De acuerdo a Cacoub y colaboradores ${ }^{29}$, entre las manifestaciones extrahepáticas de la infección crónica por VHB, la glomerulonefritis y la crioglobulinemia corresponden al $3 \%$ y al $2 \%$ de las mismas, respectivamente. En nuestro caso el paciente contaba con un diagnóstico previo de hepatitis $B$ realizado 5 años antes de la consulta detallada en el presente trabajo, por la cual recibió tratamiento sintomático y constatándose en la misma solamente positividad para Ac$\mathrm{HBc}$, interpretados como de "exposición al virus". Estudios llevados a cabo in vitro han mostrado que la IgM, cadenas kappa y factor reumatoideo de los pacientes con crioglobulinemia tipo II son propensos a unirse a la fibronectina del mesangio glomerular con mayor afinidad que la lgM de pacientes con macroglobulinemia de Waldenström o la IgM de pacientes con artritis reumatoidea ${ }^{30}$.

Antes de la asociación de la CM con VHC, muchos pacientes eran tratados satisfactoriamente con prednisona, ciclofosfamida y clorambucil ${ }^{20}$. La crioglobulinemia de difícil control se trata con terapia inmunosupresora, esteroides y plasmaféresis. En estos casos las manifestaciones cutáneas y artralgias responden rápidamente, pero la evolución de la enfermedad renal es más impredecible y depende primariamente de su cronicidad. La nefropatía aguda responde bien a este tratamiento agresivo ${ }^{31,32}$. Actualmente la mayoría de los pacientes con CM asociada a VHC son tratados con agentes anti-virales, como el rituximab y el IFN pegilado ${ }^{33,34}$.

El rituximab ha sido usado satisfactoriamente para el manejo de la CM tipo II con o sin evidencia de infección por $\mathrm{VHC}^{35,36}$. Finalmente, el compromiso renal de las $\mathrm{CM}$ ha sido tratado con diálisis y trasplante, habiendo sido reportada la recurrencia en el injerto ${ }^{37}$. 


\section{Bibliografía}

1-Brouet JC, Clouvel JP, Danon F, Klein M, Seligmann M. Biologic and clinical significance of cryoglobulins. Am J Med 1974,57:775-88

2-Jennette JC, Falk RJ, Andrassy K, Bacon PA, Churg J, Gross WL, et al. Nomenclature of systemic vasculitides: the proposal of an international consensus conference. Arthritis Rheum 1994;37:187-92

3-Landau DA, Scerra S, Sene D, Resche-Rigon M, Saadoun D, Cacoub P. Causes and predictive factors of mortality in a cohort of patients with hepatitis $C$ virus-related cryoglobulinemic vasculitis treated with antiviral therapy. $J$ Rheumatol. 2010;37:615-21

4-Mazzaro C, Tulissi P, Moretti M, Mazzoran L, Pussini E, Crovatto M, et al. Clinical and virological findings in mixed cryoglobulinaemia. J Intern Med 1995;238:153-60

5-Monti G, Galli M, Invernizzi F, Pioltelli P, Saccardo F, Monteverde A, et al. Cryoglobulinemia: a multi-centre study of the early clinical and laboratory manifestations of primary and secondary disease. Q J Med 1995;88:115-26

6-Ramos-Casals M, Font J. Mixed cryoglobulinemia. Essential?. Med Clin (Barc) 1999;113:577-8

7-Frankel AH, Singer DR, Winearls CG, Evans DJ, Rees AJ, Pusey CD. Type II essential mixed cryoglobulinemia: Presentation, treatment and outcome in 13 patients. Q J Med 1992;82:101-24

8-Ferri MC, Zignego AL, Pileri SA. Cryoglobulins (review). J Clin Pathol 2002;55:4-13

9-Ferri C, Sebastiani M, Giuggioli D, Cazzato M, Longombardo G, Antonelli A, et al. Mixed Crioglobulinemia: demographic, clinical, and serological features, and survival in 231 patients. Sem Arthritis Rheum 2004;33:355-74

10-Damoiseaux J. The diagnosis and classification of the cryoglobulinemic syndrome (review). Autoimmunity Reviews 2014;13:359-62

11-Lamprecht P, Gause A, Gross WL. Cryoglobulinemic vasculitis (review). Arthritis Rheum 1999;42:2507-16

12-Ferri C, Antonelli A, Mascia MT, Sebastiani S, Fallhai P, Ferrari D, et al. B-cells and mixed cryoglobulinemia. Autoimmun Rev 2007;7:114-20

13-Retamozo S, Brito-Zerón P, Bosch X, Stone JH, Ramos-Casals M. Cryoglobulinemic disease. Oncology (Williston Park). 2013 Nov;27(11):1098-1105

14-AntonelliA, FallahiP, Ferrari SM, CorradoA, Sebastian M, GiuggioliD, et al. Parallel increase of circulating CXCL11 and CXCL10 in mixed cryoglobulinemia, while the proinflammatory cytokine IL-6 is associated with high serum Th2 chemokine CCL2. Clin Rheumatol. 2013;32:1147-54

15-Sabbatini A, Dolcher MP, Marchini B, Chimenti D, Moscato S, Pratesi F, et al. Clin Exp Rheumatol 1997;15:655-8

16-Meltzer M, Franklin EC, Elias K, McCluskey RT, Cooper N. Cryoglobulinemia: A clinical and laboratory study. II. Cryoglobulins with rheumatoid factor activity. Am J Med 1966;40:837-56

17-Tedeschi A, Baraté C, Minola E, Morra E. Cryoglobulinemia. Blood Reviews 2007;21:183-200

18-D'Amico G. Renal involvement in hepatitis C infection: cryoglobulinemic glomerulonephritis induced byhepatitis $C$ virus. Kidney Int 1998;54:650-71

19-Hirahashi J, Kuramochi S, Konishi K, Chikaraishi A, Takase O, Hayashi M, et al. Glomerulonephritis with microtubular deposits associated with cryoglobulinemia and chronic active hepatitis. Pathol Int. 2002 Jul;52(7):483-7

20-Tarantino A, De Vecchi A, Montagnino G, Imbasciati E, Mihatsch MJ, Zollinger HU, et al. Renal disease in essential mixed cryoglobulinemia: Long-term follow-up of 44 patients. Q J Med 1981;50:1-30

21-Cordonnier D, Martin H, Groslambert P, Micouin C, Chenais F, Stoebner P. Mixed IgG-lgM cryoglobulinemia with glomerulonephritis: Immunochemical, fluorescent and ultrastructural study of kidney and in vitro cryoprecipitate. Am J Med 1975;59:867-72

22-D'Amico G, Colasanti G, Ferrario F, Sinico RA. Renal involvement in essential mixed cryoglobulinemia. Kidney Int 1989;35:1004-14

23-Choo QL, Kuo G, Weiner AJ, Overby LR, Bradley DW, Houghton M. Isolation of a cDNA clone derived from a blood-borne non-A, non-B viral hepatitis genome. Science 1989;244:359-62

24-Pascual M, Perrin L, Giostra E, Schifferli J. Hepatitis C virus in patients with cryoglobulinemia type II [letter]. J Infect Dis 1990;162:569-70

25-Misiani R, Bellavita P, Fenili D, Borelli G, Marchesi D, Massazza M, et al. Hepatitis $C$ virus infection in patients with essential mixed cryoglobulinemia. Ann Intern Med 1992;117:573-7

26-Galli M. Viruses and cryoglobulinemia. Clin Exp Rheumatol 1995;13:63-70

27-Agnello V, Chung RT, Kaplan LM. A role for hepatitis C virus infection in type II cryoglobulinemia. N Engl J Med 1992;327:1490-5

28-Levo Y, Gorevic PD, Kassab HJ, Zucker-Franklin D, Franklin EC. Association between hepatitis B virus and essential mixed cryoglobulinemia. N Engl J Med 1977;296:1501-4

29-Cacoub P, Terrier B. Hepatitis B-related autoimmune manifestations. Rheum Dis Clin North Am 2009;35:125-37

30-Fornasieri A, Armelloni S, Bernasconi P, Li M, Septis C, Sinico R, et al. High binding of IgMk rheumatoid factor from type II cryoglobulins to cellular fibronectin. Am J Kidney Dis 1996;27:476-83

31-Ben-Bassat M, Boner G, Rosenfeld J, Pick Al, Kahana M, Hazaz B, et al. The clinicopathologic features of cryoglobulinemic nephropathy. Am J Clin Pathol 1983;79:147-56

32-Valbonesi M, Montani F, Mosconi L, Florio G, Vecchi C. Plasmapheresis and cytotoxic drugs for mixed cryoglobulinemia. Haematologia (Budap) 1984;17:341-51

33-Poynard T, Bedossa P, Chevallier M, Mathurin P, Lemonnier $C$, Trepo $C$, et al. A comparison of three interferon alfa- $2 \beta$ regimens for the long-term treatment of chronic non- $A$, non- $B$ hepatitis. $N$ Engl J Med 1995;332:1457-62 
34-Cobelo C, Sola E, López V, Gutiérrez de la Fuente C, Hernández Marrero D. Crioglobulinemia mixta en una paciente sometida a transplante renal. Nefrología 2010; 30:480-1

35-Martina MN, Solé M, Massó E, Pérez N, Campistol JM, Quintana LF. Mixed cryoglobulinaemia not related to hepatitis C virus, mesangiocapillary glomerulonephritis and lymphoplasmocytic lymphoma. Nefrologia 2011;31:743-6

36 Zaja F, De Vita S, Mazzaro C, Sacco S, Damiani D, De Marchi G, et al. Efficacy and safety of rituximab in type II mixed cryoglobulinemia. Blood 2003;101:3827-34

37 Hiesse C, Bastuji-Garin S, Santelli G, Moulin B, Cantarovich M, Lantz O, et al. Recurrent essential mixed cryoglobulinemia in renal allografts. Report of two cases and review of the literature. Am J Nephrol 1989;9:150-4 